\title{
DE LA NUMÉRATION DES GERMES DU LAIT PAR LA MÉTHODE DE SKAR
}

\author{
par M. M.-F. CARRIEU
}

de Montpellier.

Nombreux sont les auteurs qui ont cherché à rendre plus pratiques et plus simples les procédés utilisés en vue de la numération des germes contenus dans un échantillon de lait. Cette opération en effet est considérée par tous comme indispensable à la surveillance du lait livré à la consommation, lait qui peut être et qui est en réalité souvent souillé par deux ordres de germes : $1^{0}$ les très nombreux saprophites banaux, non dangereux par eux-mêmes pour l'homme, mais amenant presque toujours une altération du produit où ils se multiplient avec une rapidité extraordinaire; le lait est alors rendu moins assimilable et, plus tard, impropre à la consommation; $2^{\circ}$ les germes pathogènes, beaucoup plus rares heureusement, qui proviennent de l'animal lorsqu'il est malade, ou, beaucoup plus fréquemment, du trayeur porteur de germes, ou encore, dans les cas de fraude, de l'addition d'une eau souillée.

Il est à peu près impossible, avec nos moyens actuels d'investigation, de mettre pratiquement en évidence les bactéries dangereuses contenues dans un échantillon et de les identifier : cela au moins avant la consommation de ce même lait, car cette recherche demande de longues heures. On se contente donc habituellement de numérer tous les germes contenus dans 1 c.c. de lait, sans préciser davantage leur nocivité, en tenant compte seulement de ce fait que, plus un lait est riche en microbes, plus il a été souillé, et, par conséquent, modifié dans ses éléments, et, en outre aussi, plus il a de chances de contenir des germes pathogènes.

Les méthodes indirectes proposées (réductasimétrie, coagulation par l'alcool ou la chaleur, etc.) ne donnent que des résultats très approximatifs dont on peut, d'ailleurs, se contenter dans un certain nombre de cas. Quant à la numération proprement dite des germes, on peut la faire soit en ensemençant une quantité connue de lait, ordinairement très diluée, dans certains milieux (gélatine, gélose, etc.), soit en com ptant directement les microbes sous le microscope après les avoir, bien entendu, colorés.

L $э$ plus ancien parmi ces derniers procédés est dû assurément à Skar, d'Oslo (1) qui le décrivit en 1912. Récemment C. Neukomm, de Lausanne (2) en a donné dans Le Lait une excellente mise au point.

Cette méthode consiste à compter les germes contenus dans une quantité donnée de lait visible dans un champ de microscope de surface eonnue: Mais d'abord quel est le matériel employé ? Il comprend : $1^{0}$ un osulaire où sont gravés 2 carrés et un cercle à centre commun, divisés en 4 par 2 axes perpendiculaires et dont les surfaces sont connues ; $2^{\circ}$ une lame porte objet qui présente une surface rectangulaire de 
$500 \mathrm{~mm}^{2}$ dont les côtés sont tracés en relief. Il faut d'abord régler la longueur du tube du microscope de telle sorte que le côté du grand carré ait $50 \mu$; sa surface sera done de $50 \mu \times 50 \mu=0 \mathrm{~mm}^{2}, 0025$ ou $\frac{1}{400}$ de $\mathrm{mm}^{2}$. La surface du cercle, 10 fois plus grande, atteindra alors $\frac{1}{40}$ de $\mathrm{mm}^{2}$ et celle du petit carré, 10 fois moindre, sera de $\frac{1}{4000}$ de $\mathrm{mm}^{2}$.

Pour colorer le lait, on en prend $5 \mathrm{cc}$. auxquels on ajoute $0 \mathrm{cc} .20 \mathrm{de}$ bleu. Nous avons employé le bleu de Borel qui nous a donné d'excellents résultats ; du reste le bleu de Piana et d'autres ont été également employés avec succès. Puis on agite et l'on en prélève avec une pipette spéciale $20 \mathrm{~mm}^{3}$ qui sont portés sur la lame, soigneusement nettoyée, auparavant, à l'alcool. L'étalement est fait aussi régulièrement que possible en inclinant diversement la préparation, puis on laisse sécher à la température ordinaire et bien à plat en mettant à l'abri des poussières atmosphériques (nous nous contentons de recouvrir la lame d'un couvercle de boite de Pétri). Notons, en passant, que la pipette doit toujours être, immédiatement après usage, lavée à l'eau distillée, puis à l'alcool à $95^{\circ}$.

Il ne reste maintenant qu'à évaluer le volume du lait étalé et vu dans un des carrés ou dans le cercle de l'oculaire. Nous savons que la surface du grand carré est égale à $1 / 400^{\mathrm{e}}$ de $\mathrm{mm}^{2}$ et que celle du rectangle gravé sur le porte-objet est de $500 \mathrm{~mm}^{2}$. On verra done, à travers ce grand carré, une certaine quantité de la surface totale du rectangle du porteobjet. Laquelle? Exactement $\frac{1}{\frac{400}{500}}$ ou $\frac{1}{200.000}$ du rectangle. Or sur ce

rectangle, il a été étalé $20 \mathrm{~mm}^{3}$, ou l $/ 50^{\mathrm{e}}$ de cc. Il est facile de comprendre qu'il y en aura 200.000 fois moins dans la surface vue à travers le grand carré de l'oculaire ou $\frac{\frac{1}{50}}{200.000}=\frac{1}{10.000 .000}$ de ce. Si donc l'on trouve en moyenne un germe dans chacun des grands carrés (a près 30 à 40 examens minutieux de la préparation), on pourra dire qu'il y a $1 \times 10.000 .000=10.000 .000$ de germes par cc. de lait étudié. Le petit earré étant 10 fois plus petit, il y en aurait 100.000 .000 et le cercle étant 10 fois plus grand, le résultat serait de $\mathbf{1 . 0 0 0 . 0 0 0 ~ d e ~ g e r m e s ~ p a r ~ c c . ~ e n ~}$ continuant à admettre qu'on a trouvé en moyenne 1 germe pour chaque champ examiné. En réalité il existe une petite cause d'erreur due à l'addition de $0 \mathrm{cc} .20$ de colorant pour $5 \mathrm{cc}$. de lait, soit une augmentation de $1 / 25$ ou $4 \%$ à laquelle il serait facile de remédier si la pipette portait au-dessus de $20 \mathrm{mmc}$. un trait supplémentaire marquant, en plus, 
0 mme. 8. C'est, comme on le voit, un petit détail de construction du matériel à modifier.

Nous avions donc par cette méthode - beaucoup plus simple en réalité que ce qu'elle paraît à la lecture de sa description - étudié une soixantaine de laits prélevés dans les divers dépôts ou laiteries de Montpollier. Nous avons ainsi obtenu des résultats variant de 160.000 germes par ce. à 39.000 .000 et même à 97.000 .000 . (A noter que ces chiffres élevés ont été trouvés le 3 et le 30 mai dans des échantillons provenant du même dépôt. Un essai de réductasimétrie a donné une réaction positive en 15 minutes. II s'agissait done d'un lait impropre à la consommation, conservé depuis la veille probablement et en période de grosse chaleur. Il est utile d'ajouter que 2 ou 3 autres échantillons prélevés au même endroit n'ont jamais montré une telle souillure). Mais la grande majorité des échantillons contenait, d'après cette méthode, de 1.500 .000 à 6.000 .000 de germes par ec.

Nous avons comparé les résultats ainsi obtenus avec ceux que nous a donnés la numération sur plaques de gélatine, en suivant la technique couramment employée en vue des analyses d'eau. Voici comment nous avons procédé : le lait est dilué à $1 / 10$ dans du sérum physiologique stérile (stérilité vérifiée du reste très souvent par culture) et une et cinq gouttes sont ensemencées dans $10 \mathrm{cc}$. de gélatine préalablement portée à $37^{\circ}$ et étalée ensuite en boîtes de Pétri. On étiquette, on laisse refroidir et on conserve à la température du laboratoire. Chaque jour, les colonies apparues sont comptées et marquées et cela jusqu'à liquéfaction ou jusqu'au 15 e jour. On rapporte ensuite les chiffres obtenus au cc. et on prend la moyenne des diverses boîtes ensemencées avec le même échantillon.

Les résultats sont loin d'être concordants. Sauf dans un seul cas, nous avons toujours trouvé des chiffres beaucoup plus forts avec la méthode de Skar. Par exemple nous ne trouvons qu'exceptionnellement 1.430.000 (Skar) et 904.160 (gélatine) ou 160.000 et 113.000 ; moins fréquents encore sont les chiffres suivants : 13.200.000 germes au cc. par la numération directe et 559.520 par culture, soit 25 fois moins. En pratique nous avons obtenu très souvent : 3.150 .000 contre 679.620 ou 2.660 .000 contre 573.300 , soit une proportion d'environ $5 / 1$.

Du reste Brew (3) a fait des comparaisons analogues entre une méthode très voisine, celle de Breed, et le comptage des colonies sur plaques d'agar laissées 5 jours à $21^{\circ}$; et il conclut que, lorsque le nombre des germes est peu élevé, le procédé direct donne des chiffres 44 fois plus forts, mais eette différence s'atténue pour arriver à notre proportion la plus fréquente de $5 / 1$, à mesure que les nombres trouvés sont plus considérables.

Pourquoi ces différences ? Pour plusieurs raisons dont deux nous paraissent primordiales : d'abord parce que la méthode des cultures ne donne que 
le chiffre des aérobies, d'où résultat plus faible ; mais surtout parce que parce procédé nous ne mettons en évidence que les colonies, c'est-à. dire les amas microbiens qui naissent d'un germe vivant, tandis que la numération directe sous le microscope nous donne indifféremment et les morts et les vivants. La preuve en est aisée à faire. Si nous pratiquons la même recherche sur le même lait avant et après son ébullition, nous trouvons sensiblement le même nombre de germes au cc. ; la petite différence qui existe est toujours en faveur du lait bouilli où il semble qu'il y ait un nombre plus considérable de germes. Mais ce n'est là qu'une apparence car, en réalité, le chauffage a fait évaporer une partie du liquide et a, par conséquent, légèrement accru le nombre des microbes par rapport au volume examiné; t tandis que le lait ensemencé a près ébullition et refroidis. sement immédiat ne donne jamais de culture, Quant aux autres imperfections, elles résultent du chiffre par lequel on est obligé de multiplier les résultats trouvés. Du reste il est possible de faire, jusqu'à un certain point, les mêmes reproches à toutes les méthodes utilisées actuellement pour numérer les germes du lait. Quoi qu'il en soit, alors que certaines sont d'une lenteur désespérante, puisqu'elles nécessitent plusieurs jours de culture par exemple, et rendent leur emploi inutilisable dans un grand nombre de cas, le procédé que nous venons d'étudier a un gros avantage : sa rapidité. Si l'on a soin de mettre les lames à l'étuve à $\mathbf{3 7 0}$ pour sécher plus vite le lait coloré, il permet, en effet, de donner en moins de 15 minutes une réponse dont on peut pratiquement se contenter. Ainsi donc - et ce seront nos eonclusions - nous avons à notre disposition, grâce à la méthode de Skar, un procédé de numération des germes du lait extrêmement rapide et assez précis. Il le sera encore davantage si l'on prend soin de se servir d'une pipette mieux graduée, comme nous l'avons déjà indiqué. Mais, nous le répétons, il ne faut lui demander aucun renseignement sur l'état de vie ou de mort des microbes, pas plus, du reste, que l'ensemencement sur gélatine ou gélose n'est capable de nous renseigner sur le nombre des germes contenus dans un échantillon de lait lorsque ils ont été préalablement tués par l'ébullition. Or il nous paraît préférable de connaître la totalité des germes qui, vivants ou morts se trouve dans le lait examiné et qui sont la conséquence des souillures dues aux manipulations qu'il a subies depuis le moment de la traite jusqu'à celui de l'examen. En tenant compte de ce fait que la méthode de Skar - comme toutes les recherches directes - donne un chiffre plus considérable que les cultures et en ne comparant les résultats obtenus qu'arec ceux de ce même procédé, on a ainsi à sa disposition une technique simple, rapide et sûre dont le besoin se faisait sentir en vue du contrôle bactériologique du lait.

\section{BIBLIOGRAPHIE}

[1] Skar, Zeitschrift für Fleisch- und Milchygiene, 1913, p. 301 et 442, et Centralblatt für Bakteriologie, 1922, p. 826. 
[2] C. Neukomm, Le Lail, 1927 , p. 728 et 843.

[3] BREW JAMEs, cité dans le Bulletin mensuel des renseignements agricoles et des maladies des plantes, 1922, p. 1483;

\title{
LA MÉTHODE SYNTHÉTIQUE DANS L'ÉTUDE DU LAIT LE LAIT AU POINT DE VUE COLLOIDAL RECHERCHES SUR LE MÉCANISIME DE L'ACTION DE LA PRÉSURE
}

\author{
par Ch. PORCHER, \\ Docteur ès sciences physiques
}

(Suite)

\section{LE CASEINATE DE CALCIUM TEL QU'IL EXISTE DANS LE LAIT}

La caséine existe dans le lait de vache à l'état de caséinate de calcium, lequel est uni colloïdalement aux phosphates de calcium pour former un complexe. Mais par la pensée nous pouvons très bien distraire ces derniers sels, isoler par conséquent le caséinate de calcium, et se demander quel peut en être le $p \mathrm{H}$, autrement dit, quelle en est la richesse en chaux ? Sera-ce un easéinate acide, donc d'un $p \mathrm{H}<7$, un caséinate alcalin, d'un $p \mathrm{H}>7$, ou un caséinate neutre ou sensiblement neutre d'un $p \mathrm{H}=7$ ou voisin de 7 .

Il faut, tout de suite, dans le cas du lait de vache dont la réaction est toujours acide, éliminer la question d'un $p \mathrm{H}$ nettement alcalin. Restent done les hypothèses d'un $p \mathrm{H}$ acide et d'un $p \mathrm{H}$ neutre, c'est-àdire d'un caséinate aux environs de 600 , entre 590 et 610 , c'est-à-dire neutre ou fort voisin de la neutralité.

Lait et complexe sont des mélanges : leur réaction à l'un comme à l'autre est une somme. - Dans l'un comme dans l'autre cas, qu'il s'agisse de la liqueur aux multiples éléments qu'est le lait ou de la solution beaucoup plus simple du complexe, il y existe des éléments d'une acidité marquée ; ce sont les phosphates bi- et monopotassiques dans le cas du lait, le phosphate mono-calcique dans le cas du complexe. S'ils disparaissent, la partie qui reste tend vers l'alcalinité.

Des expériences de dialyse effectuées sur le lait ou le complexe, tel qu'il sera préparé plus tard, nous montrent en effet une marche non douteuse vers l'alcalinité de la liqueur restée dans le dialyseur.

Tel lait témoin dont le $p H$ est de $6,81,-p H$ élevé pour un lait, voit ce $p \mathrm{H}$ monter à 7,05 par dialyse ; dans tel autre, le $p \mathrm{H}$ passe de 6,98 . à 7,20 . Le $p H$ de tel complexe passe par dialyse de 6,91 à 7,12 .

Il n'y a pas lieu d'insister davantage ici sur les effets de la dialyse sur le lait et sur le complexe ; la place en est indiquée lorsque nous étudierons le mécanisme de l'emprésurage. Mais nous avons voulu retenir des quelques chiffres donnés que la dialyse a pour but de faire tendre 